\title{
Apropiación de las ideas modernas en los discursos de la Educación Física en el ámbito escolar de la ciudad de Medellín 1812-1899
}

\author{
Appropriation of the modern ideas in the speeches of the physical education in the school area of the city of Medellín \\ 1812-1899
}

\section{Carmen Emilia García}

\begin{abstract}
Habremos perdido la memoria de nuestro encuentro...y sin embargo nos reuniremos, para separarnos y reunirnos de nuevo, allí donde se reúnen los hombres muertos: en los labios de los vivos.

Samuel Butler
\end{abstract}

\section{Resumen}

Este ejercicio investigativo constituye un acercamiento al análisis de los discursos de la Educación Física del siglo XIX en dos vías:

Una que parte de la visión de cuerpo biológico, la cual hemos denominado "desciframiento o presunción científica del cuerpo" que obedece al propósito de interrogar el cuerpo de manera científica, acorde con las ideas del proyecto de la Modernidad. En esta visión de cuerpo biológico se identifican a su vez dos perspectivas del conocimiento de los seres vivos: una, fisiología médica donde el individuo es objeto susceptible de analizarse funcionalmente, es una integración funcional de órganos, cuyos elementos constituyentes pueden inventariarse en forma precisa y agregarse y desagregarse según los intereses de la Medicina y cuya funcionalidad interna le permite independencia del medio. La otra perspectiva que identifica a los seres vivos es la denominada fisiología biológica. Allí su base de trabajo es el individuo como parte de una población en interacción e interdependiente con el ambiente y éste no pervive al margen del medio que garantiza precisamente su funcionalidad. La vida se entiende entonces como proceso, es decir, no puede ser un sistema cerrado. Los seres vivos se le presentan a la fisiología biológica como la sede de un triple flujo: de materia, de energía y de información. Las estructuras y funciones del cuerpo interactúan con el medio, a partir de las cuales ellas son modificadas, lo que a su vez garantiza la supervivencia del individuo y de la población.

Palabras clave: Ideas modernas, discurso, educación física, ámbito escolar, presunción científica del cuerpo y fisiología biológica.

\section{Abstract}

This paper is a report of the discourse analysis about the Physical Education during the 19th century there is two routes:

One that comes from the vision of biological body, which we have named a "deciphering or scientific presumption of the body". It has the intention of questioning the body in a scientific way, in accordance to the ideas of the project of Modernity. In this vision two perspectives of knowledge of the alive (vivacious) beings: are identified one, medical physiology where the individual is an object capable of being analyzed functionally, it is a functional integration of organs, in which elements can be refered to in a precise form and they can be integrated and disintegrated according to the interests of Medicine and whose (which) functionality enables independence. Another perspective that identifies living beings is the so called biological physiology. Life is the understood as a process, that is, it can not be such as a closed system living beings are presented to biological physiology as the place for a triple flux: matter, energy and information. The structures and functions of the body interact with the environment and which modifies for to ensure the survival of individual and people.

Key words: $\quad$ Modern Ideas, speech, physical education, school area, scientific presumption of the body and biological physiology.

Fecha de recepción: 5 de octubre de 2007.

Fecha de aceptación: 9 de noviembre de 2007.

* Licenciada en Educación Física y profesora de la Universidad de Antioquia. 


\section{Introducción}

La vida se entiende como proceso, es decir, no puede ser un sistema cerrado. Los seres vivos se le presentan a la fisiología biológica como la sede de un triple flujo: de materia, de energía y de información. Las estructuras y funciones del cuerpo interactúan con el medio, a partir de las cuales ellas son modificadas, lo que a su vez garantiza la sobrevivencia del individuo y de la población.

La otra vía para el acercamiento al análisis de los discursos de la educación física es la visión de cuerpo en tanto unido al alma, la hemos llamado "persuadir el cuerpo para unirlo al alma", sobre la base de la relación cuerpo-alma que emerge de los discursos escolares.

En este sentido, la educación es pensada en términos de desarrollo y cultivo de las facultades físicas, intelectuales y morales, en tanto que la unión de las tres facultades, proporciona a los individuos "armonía y perfección”. El vínculo cuerpo-alma se cruza en los discursos de los tres modos o facultades de ser del hombre, y así se estructura un saber sobre el cuerpo que pretende armonía y perfección en las formas de ser de los individuos.

Bajo estas dos visiones, la Educación Física trató de impartir una educación al cuerpo soportada en el conocimiento moderno de la época, fundamentado en los nuevos conocimientos, en las nuevas ideas, en la promesa de la felicidad, la idea de libertad, la noción de civilidad, progreso e ilustración.

\section{Desciframiento o presunción científica del cuerpo}

Estas ideas modernas sobre conocimiento fisiológico del cuerpo tanto en la concepción médica como en la bio-

56 lógica las encontramos en la producción de discursos del ámbito escolar de la educación física del siglo XIX en Medellín de manera dispersa, fragmentada, desordenada, superpuesta y discontinua. Abordar un conocimiento en estas condiciones puede constituir relaciones poco estrictas, pero no son conocimientos acumulados los unos junto a los otros, sino que construyen discursividades que pueden ser coherentes o no. Las discursividades impartidas en el ambiente institucional educativo de Medellín hacen visible un interés por interrogar el cuerpo de manera científica.

¿A qué obedece el privilegio de interrogar el cuerpo de manera científica en el ámbito escolar? En el proyecto moderno el fin de la ciencia era "la armonía de una sociedad perfecta, universal, compuesta de científicos de- dicados a sus labores puras; $y$ a la vez la promesa de una explotación racional ilimitada de los recursos naturales, gracias a las técnicas maquinistas de la misma Ciencia.

Esta se había proyectado en los silenciosos laboratorios, reproduciendo y perfeccionando el sistema global -abriendo el futuro-, gracias a la enseñanza pública, gratuita, obligatoria y a cargo del Estado, fiel servidor de la Ciencia. Así, la escuela trasmitía sus rudimentos a los niños, sus métodos y sus bases teóricas, con el abnegado fin de que todo el mundo "se apropie del proyecto de la modernidad, compuesto de individuos libres, autónomos, responsables y, desde luego muy científicos" (Duque, 2000: 49).

¿Cuáles son los discursos que educan en el desciframiento o presunción científica del cuerpo a partir del conocimiento de la fisiología médica? La fisiología es delineada desde la patología, y el medio ambiente es un agresor contra el cual debe defenderse el individuo. Esta visión fisiológica media entre el interés del ámbito escolar y las políticas estatales y sociales, cuyas motivaciones más significativas fueron la consolidación de la nación y la formación del ciudadano. Es sobre esta visión fisiológica, entre otras, en las que puede desarrollarse el Estado-nación. Tanto para el ámbito escolar como para las políticas estatales, la salud es entendida como sanar la enfermedad, aliviar el dolor y evitar el contagio. Relación que obedece normas nacientes de higiene pública formuladas por José Celestino Mutis, quien estaba al tanto del devenir científico europeo, y que se pueden resumir en deseo de prevenir la enfermedad, el interés de propiciar el estudio de las características de la sociedad, como las tasas de natalidad o las afecciones que determinan la salud de las poblaciones y el desarrollo de una intervención en los modos de vida, el hábitat, el espacio urbano. Políticas encaminadas a promover el bienestar social, sanar la ciudad y reducir las condiciones patógenas del medio de la vida.

La escuela pública intenta, a través de su proyecto educativo moderno, transformar a los habitantes de Medellín en ciudadanos civilizados e involucrados en una dinámica constante de producción. Se imponen unos nuevos ritmos sociales vinculados a la idea de progreso pensado en línea ascendente hacia el desarrollo, al avance científico que facilitará la llegada a un futuro mejor.

La escuela moderna se organiza como institución y está encargada de difundir en todos los rincones territoriales del Estado los objetivos que este quería alcanzar de los pobladores (ciudadanos con una ética del bien y de la felicidad común, disciplinados, ordenados, racionales, productivos, civilizados y sometidos a las leyes sociales). 
De ahí que no era posible pensar un mundo moderno, racional y ordenado mientras estuviera poblado de seres malolientes, sucios, que desbocaban sus pasiones, sus apetitos y sus instintos. La urgencia radicaba en crear 'cuerpos de ciudadanos', educar cuerpos colectivos aptos para vivir en sociedad, cuerpos bellos tal vez regidos por la medicina, la psiquiatría, la escuela, la educación física y la alimentación 'elaborada', racionalizada y sometida a controles médicos y nutricionales". La relación cuerposociedad está así mediada por la imagen del cuerpo sano. La salud se empieza a ver como una de las responsabilidades del individuo. Pero la educación de este individuo es ahora responsabilidad del Estado que debía esforzarse por garantizar la conservación, el ejercicio y el equilibrio de la soberanía del ciudadano.

Ahora, la fisiología biológica educa en el desciframiento o presunción científica del cuerpo y de la cual los siguientes discursos darán cuenta en los términos que Foucault llama juegos de relaciones y de coincidencias.

\section{Juegos de coincidencia a partir del vínculo del cuerpo con las funciones de la razón y el pensamiento}

El primer juego de coincidencias se da entre el desarrollo del sistema de la locomoción y las funciones de la conciencia. Por medio del movimiento moderado, los músculos se desarrollan apropiadamente y con la acción del pensamiento. Cuando la educación del cuerpo atiende tanto al desarrollo del movimiento físico como a las facultades intelectuales, se preocupa por el conveniente equilibrio de las fuerzas vitales.

En un segundo sentido, el juego de coincidencias se da entre los sistemas respiratorio, circulatorio y nervioso con el pensamiento, mediado por la calidad del aire que respira el cuerpo en movimiento. Cuando el cuerpo respira bastante aire libre, la sangre circula y vigoriza el cerebro. El juego de coincidencias así planteado alcanza un efecto sobre el ánimo y sobre "mucha parte de la buena situación moral, de la habitual benevolencia y de ese sentimiento de reverente gratitud que nos penetra a veces, depende del aire atmosférico" (Jacob, 1999). Un tercer sentido de este juego de coincidencias se muestra entre los sistemas nervioso y respiratorio con los sentimientos, a partir de la influencia benigna de la luz del sol que recibe el cuerpo en movimiento.

\section{Juego de relaciones a partir del vínculo del cuerpo con las condiciones de la Naturaleza}

La organización moderna "concibe al ser vivo inserto en la naturaleza y establece relaciones variadas con ella”.
Para que un ser se mantenga vivo, para que respire y se alimente, es necesario que se establezca un acuerdo entre los órganos encargados de estas funciones y las condiciones exteriores. Es necesario que la organización reaccione ante lo que Lamarck llama las "circunstancias". Por circunstancias se entiende los hábitat de la tierra o del agua, los suelos, los climas y las otras formas vivas del entorno de los organismos; en suma, toda la diversidad de los medios en que habita" (Jacob, 1999: 89). Así, someter el cuerpo a cambios bruscos de temperatura tiene como beneficio el fortalecerlo para soportar las enfermedades.

\section{Juego de relaciones a partir de la organización del cuerpo con el movimiento}

$\mathrm{El}$ organicismo es tributario directo del mecanicismo. El mecanicismo traduce una actitud permanente del viviente humano ante la vida. El hombre es el viviente separado de la vida por la ciencia e intentando alcanzar la vida a través de la ciencia. La ciencia le permite descubrir la estructura mecánica interna del cuerpo humano para hacerla accesible a la observación directa, es decir, a los ojos. Es la esencia del mecanicismo considerar aislable y reparable cada una de las piezas, léase órganos, de una máquina y tales son las raíces del organicismo: el ser vivo es parcelizable y el desorden es localizable. Dinámicas vitales del cuerpo: en el ámbito escolar de los discursos de la educación física decimonónica, el cuerpo descrito desde la dimensión biológica preocupada por su naturaleza física, prescribe que el institutor debe fundamentar sus conocimientos en el saber fisiológico, que estudia la dinámica de las propiedades vitales de un cuerpo organizado. Este saber da lugar a la división del organismo en dos sistemas: el sistema vegetativo y el sistema animal. El sistema vegetativo hace parte de la estructura interna del cuerpo o invisible del cuerpo, que está nominado por las funciones de la digestión, la respiración, la circulación de la sangre y las vísceras; a su vez este sistema, desempeña funciones de agente central en el organismo. Los movimientos producidos por estas funciones se dan por acción natural del cuerpo. En tanto que el ser viviente se concibe en relación con la Naturaleza, se ve reflejado en ella y se asemeja a la vitalidad e independencia de la naturaleza. En cuanto al sistema animal, que a su vez hace parte de la estructura externa o visible del cuerpo, nominado por las funciones de los nervios, las sensitivas y de la locomoción. Las funciones a su vez desempeñan acciones sobre la circunferencia del cuerpo. Los movimientos en 
el sistema animal se producen por acción racional del cuerpo.

En tanto que se concibe aquí la naturaleza como un objeto extraño e incomprensible y cuyas leyes procura penetrar paso a paso mediante la observación, la estructura visible de los seres vivos se convierte, entonces, en objeto de análisis y clasificación a partir de la observación. El cuerpo es desarticulado en un complejo funcional de órganos, localizables en sistemas o aparatos, sometido a una razón analítica que es apropiada por los discursos de la educación física, los cuales imparten técnicas y prácticas de movimientos; planifican y administran las posibilidades de utilización y apropiación del cuerpo. Con estas posibilidades, el cuerpo se "beneficia" porque adquiere desarrollo físico y salud. De este modo, los movimientos que tienen la intención de educar el cuerpo, asumen una acción directa sobre las funciones orgánicas del cuerpo tales como la "nerviosidad", la respiración, la circulación, la secreción y la digestión, y el desarrollo de la locomoción. Los movimientos prescritos por el ámbito escolar son la carrera, el salto, la andadura o marcha, la natación y el baile. A través de estos movimientos y de su relación con la funcionalidad de los órganos, la escuela imparte cierto tipos de técnicas, que permiten a los individuos efectuar con ayuda de otros algunas operaciones sobre su cuerpo y sus conductas; $y$ de esta manera producir en ellos una transformación, una modificación, y alcanzar cierto estado de perfección, de fuerza, de desarrollo físico (Foucault, 1999: 228). El cuerpo, entendido así, es idóneo para efectuar sobre él instrucciones específicas que tienen su orden, su tiempo, sus condiciones.

\section{Persuadir el cuerpo para unirlo al alma}

Educación del cuerpo encaminada hacía el conocimiento del cuerpo y su vínculo con el alma. La educación es pensada en términos de desarrollo y cultivo de las facultades físicas, intelectuales y morales. En tanto que la unión de las tres facultades proporciona a los individuos "armonía y perfección".

La educación de la escuela debe pues dirigirse en tres líneas distintas de ejercicio: físico, intelectual, y moral; y con la certidumbre de buenos resultados en todas tres, aunque frecuentemente no se les descubran. Es seguro que la actividad física vigoriza el cuerpo, y que la prudente instrucción sobre asuntos bien escogidos expone gradualmente el espíritu del niño, y que será virtuoso aquel a quien se haya acos- tumbrado a obrar en obediencia a la virtud (Pedro Felipe Montau, 1875: 255).

La armonía de estas facultades tiene como objeto principal la formación de hombres sanos de cuerpo y espíritu, capaces de ser ciudadanos de una sociedad republicana. He aquí, entonces, que la educación se preocupa por formar buenos ciudadanos, rectos moralmente, con carácter fuerte y a la vez propiciar el desarrollo de las ciencias y la renovación de las sociedades (Periódico de la Escuela Normal, 1876). El proyecto moderno veía en la educación la oportunidad para la modernización intelectual y moral de los individuos y se dio a la tarea de crear una sociedad más racional y científica, empeño que se revertiría en la formación de hombres modernos racionales, científicos, útiles a la sociedad, conocedores del valor del trabajo.

A partir de esta tentativa moderna de armonía entre las facultades, encontramos asociadas a las facultades físicas, la salud, la agilidad y el vigor; a las facultades morales, el sentimiento religioso, la fuerza de alma, la prudencia, la templanza; el amor al orden, a la justicia y sus semejantes, y a las facultades intelectuales, la atención, el juicio, la memoria, el raciocinio y la imaginación. El desarrollo y cultivo de las facultades físicas, morales e intelectuales en el ámbito escolar de los discursos de la Educación Física, está impregnado de la visión del cuerpo en relación con el alma. En cada una de las facultades se dan estos vínculos en correspondencia con el comportamiento y la conducta del cuerpo individual y social en aspectos como la enfermedad, la higiene, la enseñanza, el movimiento, los juegos, los placeres, la nutrición y la imaginación.

La armonía en este contexto se refiere a la relación posible entre la facultad física, es decir, el cuerpo y sus manifestaciones, con las facultades del alma, como la razón, la moral, el carácter, el espíritu, la voluntad, la virtud y la imaginación. La relación es en doble vía, el alma afecta al cuerpo y el cuerpo afecta el alma. La educación física del cuerpo en el ámbito escolar del siglo XIX, se vale del modo de ser físico, moral e intelectual; con la determinación de persuadir el cuerpo para unirlo al alma, a saber:

\section{El modo de ser físico}

¿Cómo el modo de ser físico, o lo que es lo mismo las facultades físicas, tienen el propósito de persuadir el cuerpo para unirlo al alma? El modo de ser físico, es entendido en los discursos de la educación física del cuerpo en relación con los movimientos corporales. La facultad 
física se define en relación con la salud, la agilidad y el vigor; paralelo a esto, los discursos vinculan esta facultad, con el modo de ser moral e intelectual, por ejemplo, cuando las condiciones de insalubridad pública y privada pueden afectar de manera negativa el poder de la voluntad, del valor, de la serenidad y de la virtud. De la misma manera, el modo de ser intelectual es afectado por el modo de ser físico, cuando este último con su movimiento corporal, favorece el desarrollo intelectual, de la memoria y combate los fenómenos de la imaginación. Se establecen en la escuela vínculos entre los diferentes modos de ser, para lograr mantener el fin último de la Educación, el cual no es otra cosa que la armonía de las facultades y sus funciones.

Un cuerpo sano es menos presa de estados afectivos contrarios a su naturaleza, un cuerpo capaz de muchas actividades tiene el poder de coordinar y asociar sus sensaciones con el entendimiento: "El cuerpo es el instrumento del alma; y se observa que ésta se resiente de todas las afecciones del cuerpo. Si el físico sufre, el moral sufre igualmente" (Archivo histórico de Antioquia, 1874).

La naturaleza del cuerpo es compleja, en tanto que existe entre sus partes una conexión tal "que las facultades del espíritu no pueden ser naturales, plenas y eficazmente ejercidas, sin la salud del cuerpo". Ahora bien, para quien posee un cuerpo en armonía con el alma, el proyecto moderno le promete felicidad, siempre y cuando se viva este precepto por todo el ciclo de la vida: mente sana en cuerpo sano. El ámbito escolar del siglo XIX es coherente con la idea moderna de progreso, en tanto vincula el cuerpo con el alma en términos esta vez cualitativos, donde las diversas direcciones que el espíritu tiene que emprender para descifrar la totalidad de la realidad y formarse una imagen correspondiente de ella, debe confluir en la educación de un ser racional constituido por la unión del cuerpo con el alma.

La noción de tiempo moderno también hace parte de las transformaciones en las relaciones que el ámbito escolar del siglo XIX quiere impartir en la educación física del cuerpo. Idea de tiempo que pretende medir desde afuera la vida y el quehacer del hombre individual y colectivo. La noción de tiempo lineal moderno se impone y trata de tomar como dominio homogéneo de referencia lo que los hombres hacen y la manera en que lo hacen. De ahí que se le asigne al trabajo del cuerpo un tiempo igual al que se le establece al trabajo del espíritu.

\section{El modo de ser moral}

El modo de ser moral en cuanto se relaciona con el sentimiento, la religión, la fuerza de alma, la prudencia, la templanza y el amor al orden, a la justicia y sus semejantes, está en correspondencia a su vez con el modo de ser físico e intelectual. A partir del modo de ser moral, la educación del cuerpo y su determinación de persuadirlo para unirlo al alma definen propósitos educativos como el de civilidad, servicios al Estado, productividad, templanza, entre otros, los cuales están en perfecta ligazón con el ideal moderno de individuo, de nación y de Estado. Ahora, ¿ cómo el modo de ser moral es afectado por el modo de ser físico? Los discursos de la educación física del cuerpo en su pretensión de mantener la armonía perfecta con el alma establecen la educación moral a través de "los movimientos dan el pleno dominio de su cuerpo, los hará más capaces de dominar su voluntad, su alma y de conservar todo el imperio sobre si mismos; y este imperio sobre si mismos los hará intrépidos en los peligros y sufridos en las penalidades de la vida" (Periódico de la Escuela Normal de Instrucción Pública).

El universo escolar se despliega en favor de un mundo inteligible y puramente conceptual, donde por medio de la imaginación y los sentidos no es posible tener la menor certeza racional. Las verdades de la naturaleza dejan de ser accesibles a la evidencia sensorial, hay que distanciarlas, someterlas a un cálculo racional. Los discursos expresan armonía entre el cuerpo y el alma, pero es inherente a ellos la certeza o la verdad de que cuando se persuade el cuerpo se está atendiendo de manera privilegiada el alma, pues existe la certidumbre de la inmortalidad del alma. "El cuerpo humano morada del alma. Grandeza de la obra divina. El alma es inmortal, el cuerpo mortal; aquella anima a éste. Mientras el alma permanece en el cuerpo hay vida; cuando es abandonado por ella, muere" (Romualdo B. Guarín, 1897).

En los discursos en el ámbito escolar para persuadir el cuerpo y unirlo al alma, es evidente la conquista de la razón en tanto provoca cambios en las maneras de pensar. ¿Cómo a través de los discursos de la educación física del cuerpo el modo de ser moral contribuye a los fines que persigue el Estado, tal y como lo piensa el proyecto moderno que llega a la ciudad de Medellín del siglo XIX?

La noción de Estado moderno alude aquí a un Estado ramificado, formando un plano de igualdad ideal en el que se conjugan y entrecruzan las clases sociales hasta formar una superficie estable. La sociedad en este contexto existe para garantizar la conservación, el ejercicio y el equilibrio de la soberanía del individuo y su punto de partida es siempre el de individuo dotado de razón y libertad intrínseca que vela por la realización de su propia felicidad. En esta idea de Estado, la escuela exhorta 
a que cada uno sea dueño de la soberanía de su obrar o dominio de sí mismo por medio de la valoración de la disciplina, el orden y dominio de la voluntad, para hacer de ellos ciudadanos libres. Cuando esta libertad se orienta a la felicidad mediante la elección racional de los medios adecuados, se enfrenta por una parte a los limitantes impuestos por otros sujetos con aspiraciones y métodos que puedan ser contrapuestos o contrarios al suyo o bien a los limitantes propios de enfrentar al mundo sólo con las propias fuerzas; así, el individuo reconoce que para alcanzar sus fines es mejor, lograr un acuerdo cooperativo con los demás hombres de manera que su propia libertad, sometida a cierto orden, se actualice y supere el ámbito meramente formal.

\section{Del modo de ser intelectual}

Ahora bien, el modo de ser intelectual desde esta misma dimensión privilegia la memoria, la atención, el juicio, el raciocinio y la imaginación, cuando la define como:

Respecto a su modo de ser intelectual no tenemos más que considerar que los movimientos dan la salud del cuerpo, y que la salud del cuerpo no puede menos de redundar en salud del espíritu, en lozanía y vigor de facultades intelectuales (Periódico de la Escuela Normal Pública, 1873).

La armonía también entre cuerpo y alma a partir del modo de ser intelectual, obedece en el ámbito escolar, a los infinitos atributos que se le atribuyen a la naturaleza -que es el fondo último de todo ser y de sí mismo-como el del pensamiento (alma) y la extensión (cuerpo), "de modo que nada espiritual puede darse sin extensión, y nada extenso sin espiritualidad" (Zweig, 1939) "La educación excesiva del intelecto es perjudicial porque postra la fuerza física y en especial el cerebro que es la que más inmediatamente se estimula con la actividad mental. El

60 movimiento excesivo no perjudica a la moralidad por su naturaleza ni a la educación física del cuerpo" (Pedro Felipe Montau, 1875: 255). Actuar sobre la imaginación se hace imperioso en un proyecto educativo moderno que tiene la intencionalidad de persuadir el cuerpo para prevenirlo contra los estímulos que provienen de los sentidos, y más bien estimular ese cuerpo en función del conocimiento racional, "pues las ideas o contenidos mentales se asocian de manera muy diferente cuando se sueña o se fantasea libremente y cuando se sigue los pasos de una demostración matemática.

El modo de ser intelectual establece vínculos con los sentidos, de manera tal que ellos estén bajo el dominio de la razón y en armonía con el alma, pues ésta es la única que puede dar gozo a los hombres. El alma desde el punto de vista intelectual puede unir a los hombres por medio de las actividades entre ellos y en interacción con los demás en cualquier género de relación social. La escuela persuade el cuerpo con actividades como la de los baños en los paseos, donde trata de armonizar y vincular cuerpo e intelecto.

Se dice que la capacidad específica del hombre es la intelectiva o racional que expresa lá potencia más elevada del alma y, "por tanto, la de mayor grado en la escala de los seres vivos, por ella el hombre es imagen de Dios". La capacidad intelectiva se manifiesta como capacidad cognoscitiva y como capacidad apetitiva. Sin embargo, el movimiento del cuerpo o la educación de éste no pueden privarse en todo momento de vincular cuerpo y alma.

Ahora, tanto el modo de ser físico, como el modo de ser moral y el modo de ser intelectual, conciben al hombre en una unidad sustancial de cuerpo y alma. Cuerpo y alma forman una unidad sustancial, lo que quiere decir que uno sin el otro son inconcebibles en el orden actual de la creación divina. Se unen sin intermedio alguno; se "impregnan" mutuamente: el alma está toda en el cuerpo entero y en cada una de sus partes imponiendo unidad a todas sus funciones. Con él y por él ejerce todas sus potencias.

Las dos visiones de cuerpo dan lugar en la escuela a la constitución de un saber que toma el papel de ciencia y que orienta la educación en ambos sentidos, donde se mezclan relaciones, diferencias, limitaciones, coincidencias, etc. Las visiones de cuerpo tanto en relación con el conocimiento biológico, como en relación con hábitos y comportamientos, las establecimos de acuerdo a la propuesta educativa de descifrar o persuadir el cuerpo en cuanto a que el movimiento corporal (el baile, la carrera, la gimnasia, el salto, la andadura y la natación) era el punto de partida para el conocimiento del individuo en las dimensiones físicas, morales e intelectuales. El conocimiento del cuerpo planteado de esta manera lo conecta con aspectos como la constitución del Estado, el conocimiento de otras ciencias diferentes a la fisiología, la estructura interna y externa del cuerpo humano, las funciones de la razón y el pensamiento, el ambiente físico escolar, las condiciones de la naturaleza.

El conocimiento que se imparte en estos discursos de la Educación Física del cuerpo no es todo lo que podemos saber de la Educación Física, pero nos da una idea bastante cercana de lo que se impuso a través de estos discursos, y este fragmento de historia hay que creerlo por que nunca lo llegaremos a conocer verdaderamente. (D) 


\section{Referencias}

Aisenstein, Ángela. (2002), La educación del cuerpo infantil en la escuela urbana poscolonial. El caso de la ciudad de Buenos Aires, 1817-1828. En www.efdeportes.com/efd15/colonia.htm

Archivo Histórico de Antioquia. (1874). Escuelas visitadas. Signatura, tomo 2795. Segovia.

Burke, Peter y otros. (1996). Formas de hacer historia. Madrid: Alianza.

Cardona Zuluaga, Patricia. (2002). La inserción de las ideas modernas en el estado soberano de Antioquia, vistas a través de los planes de instrucción pública de los liberales radicales, los conservadores del estado soberano y la regeneración, 1870-1890. Un estudio comparativo. Tesis. Medellín: Universidad Nacional de Colombia.

Canguilhem, Georges. (1985, junio). El conocimiento de la vida. La teoría celular, máquina y organismo. En revista Sociología, 8 y 9.

Cassirer, Ernst. (1981). La filosofía de la ilustración. México: Fondo de Cultura Económica

Centro de Documentación Facultad de Educación Universidad de Antioquia:

- Educación Física de la infancia. (1841). Diversas opiniones. Signatura Doc. 565 C. 153 PS. Folio 16. Bogotá.

- Elementos de Pedagogía. (1893). Ciencias de la Educación. Capítulo III. Luis y Martín Restrepo M. Signatura 10180c 218pz, p. 171. Bogotá.

- Folio 1833.4967. Bogotá.

- Gaceta de la Nueva Granada. (1847, 28 de noviembre).5750C 56Ro.

- Manual. (1846). Capítulo 2. Signatura 10133e56Ro. Folio 35. Bogotá.

- Revista de Instrucción Pública. Ricardo Rubio. (1898).Signatura F5678, p. 254. Bogotá.

- Revista de Instrucción Pública. (1898). Ricardo Rubio. Signatura F5678. Folio 254. Bogotá.

- Revista de Instrucción Pública. (1893). Signatura 5634c115pz. Folio 43.

- Revista de Instrucción Pública. (1893, enero). Tomo 10, n. ${ }^{\circ} .54$. Folio 60-61. Bogotá.

- Revista de Instrucción Pública. (1894). Signatura 277, p. 74. Bogotá.

- Sección científica. (1893). Higiene de las escuelas. F. del Valle Atiles. Signatura 7337 c 117 pz. Bogotá.

Cullen, Carlos. (1999, marzo). Cuerpo y sujeto pedagógico: de malestares, simulaciones y desafíos. Revista digital, 4(13). Buenos Aires.

Delaporte, Francois. (2002). La Filosofía de los acontecimientos. Medellín: Universidad de Antioquia.

Duque, Félix. (2000). Filosofía para el fin de los tiempos. Madrid: Akal.

Feher, Michel y otros. (1991). Fragmentos para una historia del cuerpo. 3 tomos. Madrid: Taurus.

Feher, Michel y otros. (1999). Arqueología del saber. 19. a edición. Madrid: Siglo XXI Editores.

Feher, Michel y otros. (1999). Nacimiento de la prisión. 29. a edición. México: Siglo XXI Editores.

Galantini, Guillermo. (2001, mayo). Cuerpo y salud en la modernidad: origen del surgimiento de la educación física. En Revista digital, 7(36). Buenos Aires.

García Gutiérrez, Carmen Emilia y otros. (2002). Discursos de la Educación Física del siglo XIX en Medellín. Medellín: Universidad de Antioquia.
Gómez, Luis Jair. (1985, julio). De la fisiología médica a la fisiología biológica. En Revista Universidad Nacional de Colombia, 19, 32 43. Sede Medellín.

Guaschi de Guerisoli, Dora. (1980). Historia de la educación física. Buenos Aires: AMIBEF.

Gutiérrez, Felipe. (1999). Un cuerpo para el alma. Frenología, fisiog nomía, craneometría en el siglo XIX en Colombia. Tesis. Medellín: Universidad Nacional de Colombia.

Herrera Beltrán, Claudia Ximena y Buitrago, Bertha Nelly. (1999, enero-agosto). El cuerpo del niño al interior de la organización temporal de la escuela primaria en Colombia, entre 1870 y 1890. En Revista Educación y Pedagogía, XI(23 y 24), 99-129.

Herrera Beltrán, Claudia Ximena. (2000). Las prácticas corporales y la educación física en la escuela primaria en Colombia entre 1870 y 1913. En Lúdica Pedagógica, 4. Bogotá: Universidad Pedagógica Nacional

Hinestroza Castaño, Mauricio. (1998). Naturaleza y Cultura. Segunda mitad del siglo XIX en el Valle de Aburrá. Tesis. Medellín: Universidad Nacional de Colombia- Corporación Autónoma Regional del Centro de Antioquia, Corantioquia.

Hobbes, Thomas. (2000). Tratado sobre el cuerpo. Madrid: Trotta.

Jacob, François. (1999). La lógica de lo viviente. Una historia de la herencia. Barcelona: Tusquets.

Jaramillo Uribe, Jaime. (2001). El pensamiento colombiano en el siglo $X I X$. Bogotá: Ediciones Uniandes, Ceso-ICANH-Alfaomega.

Larrosa, Jorge, ed. (1995). Escuela poder y subjetivación. Madrid: La Piqueta.

Latorre Mendoza, Luis. (1934). Historia e historias de Medellín. Siglos XVII XVIII y XIX. Medellín: Imprenta Oficial.

Le Bretón David. (1990). Antropología del cuerpo y modernidad. Buenos Aires: Nueva Visión

Le Goff, Jacques. (1995). Pensar la historia. Barcelona: Altaya.

Maffesoli, Michel. (1997). Elogio de la razón sensible. Barcelona: Paidós.

Melo, Jorge Orlando. (2001) Espacio e historia en Medellín. Bogotá: Biblioteca Luis Ángel Arango. En http://www.lablaa.org/blaavirtual/letra-m/medellin/espaci1.htm.

Paláu C, Luis Alfonso. (1984). Algunos problemas en torno a la historia de la biología. En Revista Sociología, 6-7, 35-44

Pedraza, Zandra. (1999). En cuerpo y alma. Visiones del progreso y de la felicidad. Bogotá: Universidad de los Andes, Departamento de Antropología.

Porter, Roy. (1996). Historia del cuerpo. En Formas de hacer historia. Madrid: Alianza.

Pregogyne, Ilya. (1997). El fin de las certidumbres. Madrid: Taurus.

Rosso, Pedro. (2002) El Pensamiento Médico del Siglo XIX: los obstáculos para la incorporación del método científico. En http:// wwww.uchile.cl/instituto/medicina/boletin/boletin36/disec3. html

Sala de Prensa Universidad de Antioquia:

- Anales de Instrucción Pública. (1885-1886). F 616.

- Boletín Oficial. (1870, 8 de enero). Signatura n. ${ }^{\circ} 373$, pp. 9-10. Medellín.

- Diario de Cundinamarca. (1875, 18 de agosto). Año 6, n. ${ }^{\circ} 1708$. Folio 950.

- El Monitor. Claudina Muneral. (1897, julio). Revista n. ${ }^{\circ}$ 5. Folio 228. Bogotá.

- Instrucción pública. (1874, 23 de mayo). Tomo V. Folio 294. 
- Periódico Escuela Normal de Instrucción Pública. (1875, 13 de marzo). Tomo VI, n. ${ }^{\circ} 219$. Folio 83 . Bogotá.

- Periódico Escuela de Instrucción Pública. (1882, 13 de marzo). Signatura Número XI, pp. 84-101. Bogotá.

- Periódico de la Escuela Normal. (1878, 14 de noviembre). Tomo 7. Folio 222. Bogotá.

- Periódico de la Escuela Normal. (1876, 22 de febrero). Folio 349. Bogotá.

- Periódico de la Escuela Normal. (1882, 27 de febrero). Signatura n. ${ }^{\circ}$, p. 66 Bogotá.

- Periódico la Escuela Normal de Instrucción Pública. (1872, 12 de octubre). Martín LLeras. Signatura Tomo III. Folio 327. Bogotá.

- Periódico la Escuela Normal de Instrucción Pública. (1871). Tomo 1. n. ${ }^{\circ}$.3. folio 39.

- Periódico la Voz de Antioquia. (1889, 22 de mayo). Época III. Serie VII. Folio 649. Medellín.

- Periódico la Escuela Normal de Instrucción Pública. (1873, abril). Tomo IV. Folio 127. Bogotá.

- Periódico la Escuela Normal de Instrucción Pública. (1871). Signatura Tomo 1. No. 3. Folio 39.

- Periódico la Escuela Normal de Instrucción Pública. (1876, 3 de junio). Tomo VII, n.os 269. 275, 286, 297. Folio 68, 69 y 71. Bogotá

- Romualdo B. Guarín. (1897, junio). Revista, 4. Folio 170. Bogotá

- Sala patrimonial. Biblioteca Pública Piloto. (1875). Elementos de higiene privada o arte de conservar la salud del Individuo. Pedro Felipe Montau, p. 255.
Patrimonio Documental Universidad de Antioquia. (1891, 28 de mayo). Anales de Instrucción Pública. Signatura, tomo 19, p. 8.

Santander, Francisco de Paula. (1820, 6 de octubre). Signatura 986.106 s 232, p. 154. Bogotá.

Sennett, Richard. (1994). Carne y piedra. El cuerpo y la ciudad en la civilización occidental. Madrid: Alianza.

Spinoza, Baruch. (1984). Ética. Grandes pensadores. Madrid: Trotta.

Veyne, Paul. (1984). Como se escribe la historia. Madrid: Alianza Editorial.

Zweig, Arnold. (1939). El pensamiento vivo de Spinoza. Madrid: Losada.

\section{Internet}

U.S. National Library of Medicine. (2002) 8600 Rockville Pike, Bethesda, MD 20894 Gallery Dream Anatomy. http://www.nlm.nih. gov/exhibition/dreamanatomy/da_g_I-B-2-01.html

La Facu.com. (2002). El significado de razón en la ética de Spinoza. http://www.lafacu.com/apuntes/filosofia/razon_en_la_etica_de_ Spinoza/default.htm.

Spinoza. (1992). Traducción por Henry Lurié, julio de 1989. Parte Cinco: Sobre La libertad humana, O El Poder Del Entendimiento. http://www.see.org/s06f.htm

Perso.canadoo.es. (2002).El sujeto ético-político. En Spinoza. http:// perso.wanadoo.es/angeljes/56/56.htm. 